\title{
Características clínicas en dentición decidua asociadas con edad y sexo en preescolares
}

\author{
Clinical characteristics in deciduous teeth associated with age and sex in preschoolers \\ Christie Geraldine Michelle Justo Pacheco ${ }^{1 \mathrm{a}}$, Tania Adriana Cosme Raymundo ${ }^{1 \mathrm{~b}}$, Rafael Morales Vadillo ${ }^{1 \mathrm{c}}$
}

\section{RESUMEN}

Objetivo: Determinar la prevalencia de las características clínicas en dentición decidua y su asociación con el sexo y la edad en niños de 3 a 5 años. Material y método: Fue un estudio de tipo observacional, analítico, transversal y prospectivo, cuya muestra consistió en 165 niños matriculados en el jardín del colegio "Niño Jesús" durante el año escolar 2018. Se realizaron todas las medidas de bioseguridad pertinentes y consentimientos informados previos. Los datos fueron recopilados en fichas clínicas de recolección de datos, se empleó la técnica observacional, estructurada y participativa, Respecto al análisis estadístico fue mediante una estadística descriptiva y prueba de Chi Cuadrado de Pearson. Resultados: fueron los siguientes: la relación molar más prevalente fue el escalón mesial con un $75.8 \%$, en cuanto al tipo de arco de Baume fue de tipo I con un $37.6 \%$, la presencia de espacios primates con un $62.4 \%$, al evaluar el resalte y sobremordida incisal se encontraron dentro de los valores normales de medición con un $84.2 \%$ y $69.1 \%$ respectivamente. No se encontraron diferencias significativas en cuanto a la relación con el sexo y la edad de la muestra estudiada ya que en el sexo el valor fue $p=0.644$ y en la edad $p=0.397$. Conclusión: la valoración individual de cada niño, se obtuvo una prevalencia de $74.5 \%$ de niños con por lo menos una característica clínica de futura predisposición de maloclusión presente en su dentición decidua, por lo cual se le confiere un alto riesgo a desarrollar una maloclusión futura en dentición permanente, y a la asociación de estas características clínicas de maloclusión con el sexo y la edad, no hubo diferencias estadísticamente significativas.

Palabras Clave: Prevalencia; Preescolar; Maloclusión; Características clínicas. (Fuente: DeCS BIREME)

\section{ABSTRACT}

Objective: To determine the prevalence of clinical characteristics in deciduous dentition and its association with sex and age in children from 3 to 5 years old. Materials and methods: It was an observational, analytical, cross-sectional and prospective study, whose sample consisted of 165 children enrolled in the garden of the "Niño Jesús" school during 2018. All related biosecurity measures and prior informed consent are applied. The data were recorded in clinical records of data collection, the observational, structured and participatory technique was used. Respect for the statistical analysis was by means of a descriptive statistic and Pearson's Chi Square test. Results: they were as follows: the most prevalent molar relationship was the mesial step with $75.8 \%$, as for the Baume arch type it was type I with $37.6 \%$, the presence of primate spaces with $62.4 \%$, when evaluating the projection and incisal overbite were found within the normal values of measurement with $84.2 \%$ and $69.1 \%$ respectively. No differences were found regarding the relationship with sex and age of the sample studied since in sex the value was $p=0.644$ and in age $p=0.397$. Conclusions: the individual evaluation of each child, obtained a prevalence of $74.5 \%$ of children with at least one clinical characteristic of future predisposition to malocclusion present in their deciduous dentition, which is why they are given a high risk of developing a future malocclusion in dentition. There were no statistically specific differences between the association of these clinical characteristics of malocclusion with sex and age.

Key words: Prevalence; preschool; Malocclusion; Clinical characteristics. (Source: MeSH NLM)

Recibido: 2 de mayo de 2020

Aprobado: 1 de julio de 2020

Publicado: 5 julio de 2020

. Universidad de San Martin de Porres. Facultad de Odontologia. Lima, Perú.
${ }^{\text {a }}$ Bachiller en Odontología
b Magister en Odontología
${ }^{c}$ Doctor en Educación
Correspondencia:

Este es un artículo Open Access distribuido bajo la licencia Creative Commons Atribución-NoComercialCompartirlgual 4.0

Correspondencia:

(ㄷ)(이()

Christie Geraldine Justo Pacheco

Correo electrónico: cym_2_11@hotmail.com

Citar como: Justo Pacheco CG. Cosme RaymundoT, Morales Vadillo R. Características clínicas en dentición decidua asociadas con edad y sexo en preescolares. KIRU. 2020; 17(3): 135-142.https://doi.org/10.24265/kiru.2020.v17n4. 


\section{INTRODUCCIÓN}

La maloclusión es una enfermedad que ocupa el tercer lugar dentro de las enfermedades bucales más prevalentes a nivel mundial, por detrás de la caries dental y la enfermedad periodontal. A su vez según la Organización Panamericana de la Salud (OPS), tiene un $70 \%$ de prevalencia en el Perú ${ }^{(1,2)}$.

El sistema estomatognático está conformado por cuatro componentes fundamentales: el periodonto, la articulación temporomandibular, la oclusión y el sistema neuromuscular; de los cuales la oclusión es la articulación que existe entre los dientes de ambas arcadas que implica tanto su morfología como sus angulaciones, influenciado por los músculos de la masticación, estructuras esqueléticas y los movimientos funcionales y parafuncionales de la mandíbula ${ }^{(3-5)}$.

Existen ciertos factores de tipo genético como algunas enfermedades heredadas de padres a hijos, distrofias a nivel óseo y muscular, alteraciones en el desarrollo fetal y por ende en la erupción dental; o de tipo ambiental como los hábitos nocivos y la perdida prematura de piezas deciduas por traumatismos o por la enfermedad de caries de infancia temprana; que alteran el sistema estomatognático del niño y condicionan a una maloclusión en su futura dentición permanente, ocasionando no solo problemas a nivel estético, sino también en la digestión y la fonética. Es en la niñez donde se desarrollan los estilos de vida de las personas y se fomentan las actitudes y buenos hábitos, por lo tanto, se hace necesario abordar este gran problema en edades tempranas ${ }^{(6-8)}$.

Algunos estudios como el de Pascual A. et $\mathrm{al}^{9} \mathrm{y}$ Aliaga A. et $\mathrm{al}^{10}$, Estrada Y. et $\mathrm{al}^{11}$ y Cañete R. et al 12 , Jerez E. et $\mathrm{al}^{13}$ y Murrieta $\mathrm{J}$ et $\mathrm{al}^{14}$; evaluaron diferentes características clínicas en dentición decidua, como la relación de las segundas molares deciduas, el tipo de arco de Baume, la presencia de espacios primates, el resalte y sobremordida incisal, entre otros factores; y determinaron que existía una alta prevalencia de dichas características, ya que estas se encontraron presentes en más de la mitad de las muestras evaluadas, por lo cual se hacía necesario mejorar la atención de los centros de salud y fomentar la aplicación de medidas preventivas tempranas a los niños.

Este trabajo de investigación fue orientado desde un enfoque preventivo sobre las características clínicas en la dentición decidua (la relación mola, el tipo de arco de Baume, presencia de espacios primates y tipo de resalte y sobremordida incisal) más prevalentes actuales en preescolares asociándolos al sexo y la edad.

\section{MATERIALES Y METODOS}

La metodología que se empleó en este trabajo de investigación fue de tipo observacional, analítico, transversal y prospectiva, la población estuvo constituída por preescolares matriculados en el año escolar 2018 en el jardín de la institución educativa particular "Niño Jesus" en el distrito de San Juan de Miraflores en Lima - Perú. El tipo de muestreo fue no probabilístico por conveniencia, siendo 165 niños evaluados siguiendo estrictamente todos los protocolos de bioseguridad y consideraciones éticas pertinentes, como son los permisos a las autoridades del colegio y el llenado de los consentimientos informados por parte de sus padres y/o apoderados, así como también siguiendo los criterios de inclusión.

La secuencia del presente trabajo de investigación fue la siguiente: el investigador previa capacitación teórica, fotográfica y clínica sobre características de signos clínicos de dentición decidua, accedió a las instalaciones del colegio mediante la autorización de la dirección del plantel y las pertinentes consideraciones éticas, para la realización del examen clínico de los niños de 3, 4 y 5 años de edad, exceptuando a aquellos que no contaban con la firma del consentimiento informado, a niños que habían tenido tratamiento de ortodoncia interceptiva previa, niños con presencia de piezas permanentes en sus arcadas y niños poco colaboradores, todos ellos considerados dentro de los criterios de exclusión.

Para la recolección de los datos se preparó un campo de trabajo en un ambiente dispuesto por las profesoras, utilizando una mesa forrada con papel kraft para la colocación de los materiales e instrumentales necesarios (espejos bucales $n^{\circ} 5$, gasas, bajalenguas, regla milimetrada y lápiz portaminas) y con las medidas de bioseguridad pertinentes (colocación de gorro, mascarillas, guantes y lentes protectores), se dio inicio a la evaluación de las características clínicas, se empleó la técnica rodilla a rodilla y con el uso de un frontoluz (Linterna frontal Energizer con 3 led) para una mejor visualización. se empezó por el lado derecho retrayendo el carrillo con ayuda de un espejo bucal para observar las caras distales de las segundas molares deciduas y determinar el tipo de relación molar (plano terminal recto, escalón 
mesial o escalón distal) ${ }^{(15,16)}$, repitiendo el mismo paso para el lado izquierdo, a continuación se evaluó la presencia de espacios de desarrollo en el sector anterior de ambas arcadas determinando así el tipo de arco de Baume (arco tipo I si hay espacios en ambas arcadas, arco tipo II si no hay espacios en ninguna arcada y arco tipo III si hay espacios solo en una arcada) ${ }^{(17,18)}$, a su vez se evaluó la presencia de espacios primates ubicados entre el incisivo lateral y el canino deciduo (maxilar superior) y entre el canino $y$ el primer molar deciduo (maxilar inferior) ${ }^{(19,20)}$, finalmente se evaluó la oclusión del área anterior, el resalte incisal (tipo normal, aumentado, nulo o invertido) con la ayuda de la regla milimetrada (metal / plástico) y el lápiz portaminas, siendo la distancia que hay entre el borde incisal del incisivo central superior hasta la cara vestibular del incisivo central inferior entre 1 a $4 \mathrm{~mm}$ (considerados valores normales) ${ }^{(21,22)}$, trazando una línea a nivel del borde incisal del incisivo superior en la cara vestibular del incisivo inferior, se midió también la sobremordida incisal (tipo normal, profunda, nula y abierta) hasta el borde incisal del incisivo central inferior entre 1 a $3 \mathrm{~mm}$ (considerados valores normales) ${ }^{(23,24)}$.

Toda la información fue registrada en fichas clínicas de recolección de datos, mediante la técnica observacional, estructurada y participativa ya que se contó con un personal de apoyo, para realizar las anotaciones, dichos datos fueron trasladados a una base de datos en el programa de EXCEL, mediante el programa estadístico de SPSS versión 25, empleando una estadística descriptiva para hallar los porcentajes, las frecuencias absolutas y relativas, medias $y$ desviación estándar; a su vez mediante la prueba estadística de Chi Cuadrado de Pearson, se determinó la asociación entre cada una de las características clínicas en dentición decidua con el sexo y la edad de todos los niños evaluados.

Este estudio contó con la autorización del Comité Revisor de Proyectos de Investigación de la Facultad de Odontología de la Universidad de San Martín de Porres mediante el ACTA N021-2018CRPI/INVE-FO-USMP y del Comité de Ética en Investigación de la Facultad de Odontología de la Universidad de San Martín de Porres con código ACTA N005-2018-CEI/INVE-FO-USMP.

\section{RESULTADOS}

La muestra estuvo constituida por 165 niños evaluados clínicamente, siendo 93 de ellos del sexo femenino (56.4\%) y 72 del sexo masculino (43.6\%); divididos en tres grupos etarios, con 23 niños $(13.9 \%)$ para el grupo de 36 a 47 meses, 52 niños $(31.5 \%)$ para el grupo de 48 a 59 meses y 90 niños $(54.5 \%)$ para el grupo de 60 a 71 meses. (Tabla

Tabla 1. Distribución muestral de características clínicas según sexo y edad en preescolares en el Jardín de La Institución Educativa Particular "Niño Jesus" en el distrito de San Juan de Miraflores Lima, 2018.

\begin{tabular}{lccccccccc}
\hline & \multicolumn{9}{c}{ EDAD (meses) } \\
& \multicolumn{3}{c}{$\mathbf{3 6}$ a $\mathbf{4 7}$} & \multicolumn{4}{c}{$\mathbf{4 8}$ a $\mathbf{5 9}$} & $\mathbf{6 0}$ a $\mathbf{7 1}$ & \multicolumn{2}{c}{ Total } \\
\multicolumn{1}{c}{ SEXO } & $\mathbf{N}$ & $\%$ & $\mathbf{N}$ & $\mathbf{\%}$ & $\mathbf{N}$ & $\mathbf{\%}$ & $\mathbf{N}$ & $\%$ \\
\hline Masculino & 10 & 13.9 & 24 & 33.3 & 38 & 52.8 & 72 & 43.6 \\
Femenino & 13 & 14.0 & 28 & 30.1 & 52 & 55.9 & 93 & 56.4 \\
Total & 23 & 13.9 & 52 & 31.5 & 90 & 54.5 & 165 & 100.0 \\
\hline
\end{tabular}

La relación de las segundas molares deciduas más frecuente fue el escalón mesial tanto para el lado derecho como en el izquierdo ya que se presentó en 125 de los casos (75.8\%), siendo también el más frecuente a la evaluación por sexo y edad con pequeñas diferencias entre grupos. Por otro lado el plano terminal de menor recuento fue el escalón distal con solo $10(6.1 \%)$ y $13(7.9 \%)$ casos tanto para el lado derecho como izquierdo respectivamente.

Teniendo en cuenta la presencia de los espacios de desarrollo en las arcadas dentales, se encontró que $62(37.6 \%)$ de los niños evaluados presentaba un arco de Baume tipo I, siendo este también frecuente en los grupos de sexo masculino 
y en el de 48 a 59 meses de edad. En los grupos de sexo femenino, de 36 a 47 y 60 a 71 meses de edad, el arco de Baume más frecuente fue el tipo II con $60(36.4 \%)$ casos. Mientras que el arco de menor frecuencia fue el tipo III con $43(26.1 \%)$ casos. A su vez, también prevalecieron las arcadas con espacios primates ya que estos fueron encontrados en 103 (62.4\%) niños, siendo altos también en los grupos por sexo y edad.

A la evaluación del resalte incisal, predominó el de tipo normal en $139(84.2 \%)$ de los casos con una medida mínima de $-2 \mathrm{~mm}$ y una máxima de $5 \mathrm{~mm}$, con una media de $1.99 \mathrm{~mm}$, siendo también alto a la evaluación por sexo y edad, mientras que el resalte incisal de menor recuento fue el invertido presente en $4(2.4 \%)$ casos. Por otro lado, la sobremordida incisal normal fue la de mayor prevalencia en los niños, presente en 114 (69.1\%) de los casos, siendo alto a su vez en los grupos por sexo y edad, la sobremordida incisal abierta no estuvo presente en ninguno de los niños evaluados ya que la medida mínima encontrada fue de $0 \mathrm{~mm}$, mientras que la máxima fue de $7 \mathrm{~mm}$ con una media de $2.42 \mathrm{~mm}$. (Tabla 2, Tabla 3 y Tabla 4)

En cuanto al análisis entre las variables características clínicas con el sexo no se encontraron diferencias significativamente estadísticas ya que el valor de $p$ fue mayor a 0.05 ; de igual forma al análisis de las variables signos clínicos de maloclusión con la edad, salvo en el grupo de sobremordida incisal donde $p$ obtuvo un valor de 0.033 , pero que no se considera significativamente estadístico debido a la gran diferencia entre casos por grupo. (Tabla 3 y Tabla 4).

Finalmente, al análisis de las características clínicas en una valoración individual de cada preescolar, se obtuvo una prevalencia de $74.5 \%$ (123 niños) con por lo menos una característica clínica de maloclusión presente en su dentición decidua.

Tabla 2. Prevalencia de características clínicas en preescolares en el Jardín de la Institución Educativa Particular "Niño Jesús", en el distrito de San Juan de Miraflores - Lima, 2018

\begin{tabular}{clcc}
\hline \multicolumn{2}{c}{ CARACTERÍSTICAS } & N & $\%$ \\
\hline $\begin{array}{c}\text { Plano } \\
\text { terminal } \\
\text { derecho }\end{array}$ & Recto & 30 & 18.2 \\
& Mesial & 125 & 75.8 \\
$\begin{array}{c}\text { Plano } \\
\text { terminal } \\
\text { izquierdo }\end{array}$ & Recto & 10 & 6.1 \\
& Mesial & 27 & 16.4 \\
& Distal & 125 & 75.8 \\
Arco de & Tipo I & 13 & 7.9 \\
Baume & Tipo II & 62 & 37.6 \\
& Tipo III & 43 & 36.4 \\
Espacios & Sin espacios & 62 & 26.1 \\
primates & Con espacios & 103 & 37.6 \\
& Normal & 139 & 62.4 \\
Resalte & Aumentado & 5 & 3.2 \\
incisal & Nulo & 17 & 10.3 \\
& Invertido & 4 & 2.4 \\
& Normal & 114 & 69.1 \\
Sobremordida & Profunda & 30 & 18.2 \\
incisal & Nula & 21 & 12.7 \\
& Abierta & 0 & 0.0 \\
\hline
\end{tabular}


Tabla 3. Prevalencia de características clínicas y su asociación con el sexo en preescolares en el Jardín de la Institución Educativa Particular "Niño Jesús", en el distrito de San Juan de Miraflores Lima, 2018.

\begin{tabular}{|c|c|c|c|c|c|c|}
\hline \multirow{3}{*}{\multicolumn{2}{|c|}{$\begin{array}{c}\text { CARACTERÍSTICAS } \\
\text { CLIINICAS }\end{array}$}} & \multicolumn{4}{|c|}{ SEXO } & \multirow{3}{*}{$\begin{array}{c}\text { Valor } \\
\mathrm{p} \\
\mathrm{Chi}^{2}\end{array}$} \\
\hline & & \multicolumn{2}{|c|}{ Masculino } & \multicolumn{2}{|c|}{ Femenino } & \\
\hline & & $\mathrm{N}$ & $\%$ & $\mathrm{~N}$ & $\%$ & \\
\hline \multirow{3}{*}{$\begin{array}{l}\text { Plano terminal } \\
\text { derecho }\end{array}$} & Recto & 12 & 40.0 & 18 & 60.0 & \multirow{3}{*}{0.847} \\
\hline & Mesial & 55 & 44.0 & 70 & 56.0 & \\
\hline & Distal & 5 & 50.0 & 5 & 50.0 & \\
\hline \multirow{3}{*}{$\begin{array}{l}\text { Plano terminal } \\
\text { izquierdo }\end{array}$} & Recto & 9 & 33.3 & 18 & 66.7 & \multirow{3}{*}{0.498} \\
\hline & Mesial & 57 & 45.6 & 68 & 54.4 & \\
\hline & Distal & 6 & 46.2 & 7 & 53.8 & \\
\hline \multirow{3}{*}{$\begin{array}{l}\text { Arco de } \\
\text { Baume }\end{array}$} & Tipo I & 28 & 45.2 & 34 & 54.8 & \multirow{3}{*}{0.924} \\
\hline & Tipo II & 25 & 41.7 & 35 & 58.3 & \\
\hline & Tipo III & 19 & 44.2 & 24 & 55.8 & \\
\hline \multirow{2}{*}{$\begin{array}{l}\text { Espacios } \\
\text { primates }\end{array}$} & $\begin{array}{l}\text { Sin } \\
\text { espacios }\end{array}$ & 29 & 46.8 & 33 & 53.2 & \multirow{2}{*}{0.528} \\
\hline & $\begin{array}{l}\text { Con } \\
\text { espacios }\end{array}$ & 43 & 41.7 & 60 & 58.3 & \\
\hline \multirow{4}{*}{$\begin{array}{l}\text { Resalte } \\
\text { incisal }\end{array}$} & Normal & 61 & 43.9 & 78 & 56.1 & \multirow{4}{*}{0.764} \\
\hline & Aumentado & 3 & 60.0 & 2 & 40.0 & \\
\hline & Nulo & 7 & 41.2 & 10 & 58.8 & \\
\hline & Invertido & 1 & 25.0 & 3 & 75.0 & \\
\hline \multirow{4}{*}{$\begin{array}{l}\text { Sobremordida } \\
\text { incisal }\end{array}$} & Normal & 54 & 47.4 & 60 & 52.6 & \multirow{4}{*}{0.332} \\
\hline & Profunda & 10 & 33.3 & 20 & 66.7 & \\
\hline & Nula & 8 & 38.1 & 13 & 61.9 & \\
\hline & Abierta & 0 & 0.0 & 0 & 0.0 & \\
\hline
\end{tabular}

Tabla 4. Prevalencia de características clínicas y su asociación con la edad en preescolares en el Jardín de la Institución Educativa Particular "Niño Jesús" en el distrito de San Juan de Miraflores Lima, 2018.

\begin{tabular}{|c|c|c|c|c|c|c|c|c|}
\hline \multirow{3}{*}{\multicolumn{2}{|c|}{ CARACTERÍSTICAS CLÍNICAS }} & \multicolumn{6}{|c|}{ EDAD (meses) } & \multirow{3}{*}{$\begin{array}{l}\text { Valor } p \\
\text { Chi }^{2}\end{array}$} \\
\hline & & \multicolumn{2}{|c|}{36 a 47} & \multicolumn{2}{|c|}{48 a 59} & \multicolumn{2}{|c|}{60 a 71} & \\
\hline & & $\mathrm{N}$ & $\%$ & $\mathrm{~N}$ & $\%$ & $\mathrm{~N}$ & $\%$ & \\
\hline \multirow{3}{*}{$\begin{array}{c}\text { Plano terminal } \\
\text { derecho }\end{array}$} & Recto & 5 & 16.7 & 11 & 36.7 & 14 & 46.7 & \multirow{3}{*}{0.782} \\
\hline & Mesial & 17 & 13.6 & 39 & 31.2 & 69 & 55.2 & \\
\hline & Distal & 1 & 10.0 & 2 & 20.0 & 7 & 70.0 & \\
\hline \multirow{3}{*}{$\begin{array}{l}\text { Plano terminal } \\
\text { izquierdo }\end{array}$} & Recto & 4 & 14.8 & 11 & 40.7 & 12 & 44.4 & \multirow{3}{*}{0.645} \\
\hline & Mesial & 18 & 14.4 & 38 & 30.4 & 69 & 55.2 & \\
\hline & Distal & 1 & 7.7 & 3 & 23.1 & 9 & 69.2 & \\
\hline \multirow{3}{*}{$\begin{array}{l}\text { Arco de } \\
\text { Baume }\end{array}$} & Tipo I & 8 & 12.9 & 23 & 37.1 & 31 & 50.0 & \multirow{3}{*}{0.73} \\
\hline & Tipo II & 10 & 16.7 & 17 & 28.3 & 33 & 55.0 & \\
\hline & Tipo III & 5 & 11.6 & 12 & 27.9 & 26 & 60.5 & \\
\hline \multirow{2}{*}{$\begin{array}{l}\text { Espacios } \\
\text { primates }\end{array}$} & Sin espacios & 9 & 14.5 & 15 & 24.2 & 38 & 61.3 & \multirow{2}{*}{0.281} \\
\hline & Con espacios & 14 & 13.6 & 37 & 35.9 & 52 & 50.5 & \\
\hline \multirow{4}{*}{ Resalte incisal } & Normal & 21 & 15.1 & 43 & 30.9 & 75 & 54.0 & \multirow{4}{*}{0.228} \\
\hline & Aumentado & 2 & 40.0 & 0 & 0.0 & 3 & 60.0 & \\
\hline & Nulo & 0 & 0.0 & 7 & 41.2 & 10 & 58.8 & \\
\hline & Invertido & 0 & 0.0 & 2 & 50.0 & 2 & 50.0 & \\
\hline \multirow{4}{*}{$\begin{array}{l}\text { Sobremordida } \\
\text { incisal }\end{array}$} & Normal & 21 & 18.4 & 29 & 25.4 & 64 & 56.1 & \multirow{4}{*}{$0.033^{*}$} \\
\hline & Profunda & 2 & 6.7 & 14 & 46.7 & 14 & 46.7 & \\
\hline & Nula & 0 & 0.0 & 9 & 42.9 & 12 & 57.1 & \\
\hline & Abierta & 0 & 0.0 & 0 & 0.0 & 0 & 0.0 & \\
\hline
\end{tabular}

${ }^{*}$ Resultado con significancia estadística, Chi Cuadrado de Pearson 


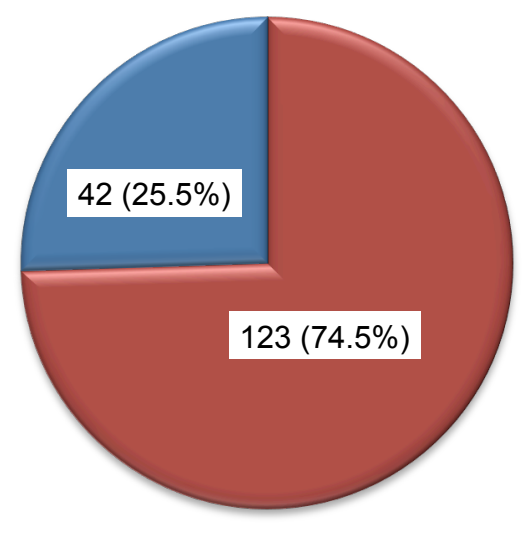

口Presentes

$\square$ Ausentes

Figura 1. Presencia de características clínicas en niños preescolares en el Jardín del la Institucion Educativa Particular "Niño Jesús" en el distrito de San Juan de Miraflores - Lima, 2018.

\section{DISCUSION}

De acuerdo a los resultados obtenidos de la muestra en el estudio, se contempla que la relación molar más frecuente fue el escalón mesial, con una prevalencia de $75.8 \%$ en ambos lados, el cual no coincide con los datos de los estudios de Pascual A. et $\mathrm{al}^{10}$, Estrada $Y$. et $\mathrm{al}^{12}$, Cañete R. et $\mathrm{al}^{13}$ y Murrieta J. et $\mathrm{al}^{15}$, porque en ellos predominó el plano terminal recto con una frecuencia de $52.5 \%, \quad 86.6 \%, 50 \%$ y $56.2 \%$ respectivamente. Caso contrario con el estudio de Jerez $\mathrm{E}$. et $\mathrm{al}^{14}$, que obtuvo un alto porcentaje de escalón mesial $(44.1 \%)$, así como de plano terminal recto $(44.1 \%)$.

Por otro lado la relación molar con menor frecuencia fue el escalón distal con $6.1 \%$ y $7.9 \%$ para el lado derecho e izquierdo respectivamente, fue similar a los estudios de Estrada $Y$. et $a^{12}$, Jerez E. et $\mathrm{al}^{14}$ y Murrieta J. et $\mathrm{al}^{15}$ con una prevalencia de $3.8 \%$, $2.9 \%$ y $7.2 \%$ respectivamente; discrepando con los estudios de Pascual $A$. et $\mathrm{al}^{10}$ y Cañete $R$. et $\mathrm{al}^{13}$ donde se obtuvo un $17.5 \%$ y $14 \%$ respectivamente para escalón mesial. Con respecto a la predicción por un determinado sexo, no hubo diferencias estadísticas significativas similar al estudio de Estrada Y. et $\mathrm{al}^{12} \mathrm{y}$ Jerez E. et $\mathrm{al}^{14}$ en los cuales al igual que en el presente estudio, hubo un número mayor de casos en niñas, mientras que en el estudio de Murrieta $\mathrm{J}$. et $\mathrm{al}^{15}$, el número mayor fue en los niños.
En relación a la edad no hubo diferencias significativas, de igual forma que en el estudio de Murrieta J. et $\mathrm{al}^{15}$, sin embargo se mostró una diferencia en el escalón distal que se presentó con un $10 \%$ en el grupo de 60 a 71 meses de edad, mientras que en los grupos de 36 a 47 y 48 a 59 meses de edad fue de $4.3 \%$ y $5.8 \%$ respectivamente.

Con respecto al tipo de arco de Baume, el de mayor frecuencia fue el arco tipo I con un $37.6 \%$ coincidente a los estudios de Murrieta J. et $\mathrm{al}^{15}$, Pascual A. et al $^{10}$ y Estrada $Y$. et al $^{12}$ ya que tuvo una prevalencia de $66.4 \%, \quad 65 \%$ y $57.7 \%$ respectivamente. No hubo diferencias en cuanto a asociación con un determinado sexo similar al trabajo de Estrada Y. et al $^{12}$ y Murrieta J. et al $^{15}$. En cuanto a la prevalencia por grupos etarios no hubo diferencias estadísticas, sin embargo se nota una pequeña inclinación por los niños de 48 a 59 meses de edad con un $44.2 \%$ para el arco tipo I, a su vez para el arco tipo II, el mayor porcentaje se encontró en los niños de 36 a 47 meses de edad con un $43.5 \%$, y para el arco tipo III, fue de $28.9 \%$ en el grupo de 60 a 71 meses de edad.

Al evaluar la existencia o no de espacios primates, se reportó una alta frecuencia de arcos con espacios primates $(62.4 \%)$, a diferencia del estudio de Estrada $Y$. et $\mathrm{al}^{12}$, que obtuvo alto porcentaje para los arcos con ausencia de espacios primates con un $63.5 \%$. En cuanto al estudio de Cañete R. et al ${ }^{13}$, existió una 
predilección por la ausencia de espacios primates en el maxilar con un $36.2 \%$ y en la mandíbula con un $43.7 \%$. No hubo diferencias estadísticas, pero se encontró una pequeña diferencia respecto al sexo femenino que presentó un $64.5 \%$ de arcos con espacios primates, resultado al similar al estudio de Estrada $Y$. et $a^{12}$ con un $40.4 \%$. En cuanto a la asociación con grupos etarios no hubo diferencias estadísticas, encontrándose en un $71.2 \%$ en el grupo de 48 a 59 meses los arcos con espacios primates, mientras que los arcos sin espacios, fueron encontrados mayormente en niños de 36 a 47 y 60 a 71 meses de edad.

Al analizar el resalte incisal se halló un alto valor de resalte incisal normal $(0-4 \mathrm{~mm})$ con un $84.2 \%$ de prevalencia que coincide con el resultado de la investigación de Cañete et al $^{13}$ que obtuvo un $57.5 \%$. En el estudio de Jerez $\mathrm{E}$. et $\mathrm{al}^{14}$ encontraron un $3.9 \%$ de resalte incisal invertido y en el estudio de Aliaga A. et al $^{11}$, un $16.4 \%$. No hubo diferencias significativas al evaluar por sexo ya que se encontró un $84.7 \%$ para el masculino y un $83.9 \%$ para el femenino. Respecto a la edad se encontró que en el grupo de 36 a 71 meses de edad un $91.3 \%$ de resalte incisal normal y un $8.7 \%$ de resalte incisal aumentado, a su vez un resalte incisal nulo fue predominante en niños de 48 a 59 y 60 a 71 meses de edad, sin diferencias estadísticas. También se halló que la media fue de $1.99 \mathrm{~mm}$ con un valor mínimo de $-2 \mathrm{~mm}$ y un valor máximo de $5 \mathrm{~mm}$.

Al determinar la sobremordida incisal se obtuvo un $69.1 \%$ para la sobremordida incisal normal (1$3 \mathrm{~mm}$ ), en comparación con el estudio de Jerez $\mathrm{E}$. et $\mathrm{al}^{14}$, que obtuvo un $39.2 \%$ para la sobremordida incisal profunda. Con referencia al sexo, se obtuvo un $75 \%$ para el masculino con sobremordida incisal normal, mientras que con un $21.5 \%$ para profunda en femenino, sin diferencias estadísticas al igual que en la asociación con la edad. Encontrándose que la media fue de $2.42 \mathrm{~mm}$ con un valor mínimo de $0 \mathrm{~mm}$ y un valor máximo de $7 \mathrm{~mm}$.

Finalmente se concluye que en cuanto al tipo de plano terminal, arco de Baume, espacios primates, resalte y sobremordida incisal, los porcentajes encontrados más altos a la evaluación individual por niño, confieren un alto riesgo en la muestra examinada para el futuro desarrollo de una maloclusión porque alrededor de más de la mitad de los preescolares presentaron signos clínicos de maloclusión en su dentición decidua de acuerdo a los parámetros establecidos de evaluación, con una prevalencia de $74.5 \%$. Por ello se hace necesaria la evaluación de niños en edades tempranas, ya que es así como podemos fomentar una buena educación bucal tanto en ellos como en sus padres o apoderados, de la mano del personal de salud y los docentes a futuro.

Contribución de autoría: CGMJP y TCR diseñaron el estudio y recogieron los datos. CGMJP,TCR y RMV análizaron los datos. La redaccion y revisión crítica fue realizada por todos los autores.

Fuentes de financiamiento: autofinanciada.

Conflicto de Interés: Los autores no declararon conflictos de interés.

\section{REFERENCIAS BIBLIOGRAFICAS}

1. Pan American Health Organization. Ten-year evaluation of regional core health data initiative. Epidemiol Bull PAHO. 2004; 25(3):1-16.

2. Orellana $\mathrm{O}$, Mendoza J, Perales $\mathrm{S}$, Marengo $\mathrm{H}$. Estudio descriptivo de todas las investigaciones sobre maloclusiones realizadas en las universidades de Lima, Ica y Arequipa. Odontol Sanmarquina. 2000; 1(5): 39-43.

3. Cuccia A, Caradonna $C$. The relationship between the stomatognathic system and body posture. Clinics (Sao Paulo). 2009; 64(1):61-66.

4. The Academy of Prosthodontics. Glossary of Prosthodontic Terms. J Prosthet Dent. 2017; 117(55): e001-e105.

5. Laganà G, Venza N, Borzabadi-Farahani $A$, Fabi $F$, Danesi C, Cozza P. Dental anomalies: prevalence and associations between them in a large sample of nonorthodontic subjects, a cross-sectional study. BMC Oral Health. 2017; 17(1):62

6. Scarpelli B, Berger S, Punhagui M, Zeri C, Ferelle $A$, Oltramari P. Evaluation of a preventive educational program for malocclusions: 7-year study. Braz Oral Res. 2016; 30(1): 1-8.

7. Kumar D, Gurunathan D. Primary Canine and Molar Relationships in Centric Occlusion in 3- to 6-yearold Children: A Cross-sectional Survey. Int J Clin Pediatr Dent. 2019; 12(3): 201-204.

8. Glick M, Williams D. Vision 2020 Think Tank A new definition for oral health. FDI World Dental Federation. 2016.

9. Pascual A, López E. Tipo de arcada y plano terminal molar de la dentición temporal y su correlación con las clases de maloclusión de la dentición permanente. Apunt Cienc Soc. 2015; 5(2): 310-315.

10. Aliaga A, Mattos $M$, Aliaga $R$, Castillo $C$. Maloclusiones en niños y adolescentes de caseríos y comunidades nativas de la amazonía de Ucayali, Perú. Rev Peru Med Exp Salud Publica. 2011; 28(1): 87-91. 
11. Estrada Yadira, Estrada Yelec, Cubero R, López D. Factores de riesgo de maloclusiones en niños de 5 años con dentición temporal. Mediciego. 2015; 21(1): 1-7.

12. Cañete $R$, Frías $O$, Osoria B. Alteraciones en la oclusión de niños en edades de 3 a 5 años. Multimed. 2013; 17(2): 1-14.

13. Jerez E, Zerpa R, Salas M, Simancas $Y$, Romero $Y$. Prevalencia de maloclusiones en niños del jardín de infancia de la escuela bolivariana "Juan Ruiz Fajardo". Act Bioclinica. 2014; 4(8): 54-69.

14. Murrieta J, Torres N, Linares C, González M, Juárez $\mathrm{M}$, Morales $\mathrm{J}$ et al. Prevalencia de planos terminales y tipo de arcada y su relevancia en la oclusión dental en un grupo de preescolares. Odonto Pediatría. 2014; 3(9): 30-35.

15. Kasparaviciene K, Sidlauskas A, Zasciurinskiene E, Vasiliauskas A, Juodzbalys $G$, Sidlauskas $M$, Marmaite $U$. The prevalence of malocclusion and oral habits among 5-7-year-old children. Med Sci Monit. 2014; 20: 2036-2042.

16. Lee $\mathrm{Y}$, Huang $\mathrm{Y}$, Chen I, Lin $\mathrm{P}$, Chuang L. Craniofacial, dental arch morphology, and characteristics in preschool children with mild obstructive sleep apnea. Journal of Dental Sciences. 2019; 15(2): 193-199.

17. Baume L. Physiological tooth migration and its significance for the Development of occlusion: II. The biogénesis of accesional dentition. J Dent Res. 1950; 29: 331-337.
18. Murrieta J, Velázquez $\mathrm{Y}$, Yáñez M, Adriano M, Joya T. Parafunctional oral habits and primary dentition characteristics in a group of preschool children from Tlaquepaque, Jalisco, Mexico. J Oral Res 2019; 8(1):50-58.

19. Pradhan M, Dhital S, Dahal S, Joshi U, Sherchan P, Pokhrel P. Occlusal characteristics including spacing among preschool children: a crosssectional study. J Chitwan Med Coll. 2019; 9(30): 28-31.

20. Sun KT, Li YF, Hsu JT, Tu MG, Hung CJ, Hsueh $\mathrm{YH}$, et al. Prevalence of primate and interdental spaces for primary dentition in 3- to 6-year-old children in Taiwan. J Formos Med Assoc. 2018 Jul; 117(7): 598-604.

21. Amores F, Moya T. Maloclusiones dentarias en el sector anterior con relación a la clase molar en estudiantes que tocan el instrumento clarinete. Dom Cien. 2017; 3(1): 462-473.

22. Ameer SAA, Al-Ansari NB, Nahidh M. Occlusal Features of Deciduous Dentition in an Iraqi Sample. Int Med J. 2020; 25(1): 347-356.

23. Gupta S, Sadana G, Mehra M, Grover R, Bhargawa A. Assessment of oclusal characteristics in primary dentition of preschool children in Amritsar, Punjab, India. Curr Trends Diagn Treat. 2018; 2(1): 15-21.

24. Maaniitty $E$, Vahlberg $T$, Lüthje $P$, Rautava $P$, Svedström A. Malocclusions in primary and early mixed dentition in very preterm children, Acta Odontologica Scandinavica. 2020; 78(1): 52-56.

Christie Geraldine Michelle Justo Pacheco
cym_2_11@hotmail.com $\begin{aligned} & \text { Tania Adriana Cosme Raymundo } \\ & \text { tcosmer@usmp.pe }\end{aligned}$

